\title{
Perpendicularly magnetized Ni/Pt (001) epitaxial superlattice
}

\author{
T. Seki $\odot,{ }^{1,2,3,{ }^{*}}$ M. Tsujikawa, ${ }^{2,4}$ K. Ito $\odot,{ }^{1,2}$ K. Uchida $\odot,{ }^{1,2,3}$ H. Kurebayashi, ${ }^{5}$ M. Shirai, ${ }^{2,4,6}$ and K. Takanashi $\circledast^{1,2,6}$ \\ ${ }^{1}$ Institute for Materials Research, Tohoku University, Sendai 980-8577, Japan \\ ${ }^{2}$ Center for Spintronics Research Network, Tohoku University, Sendai 980-8577, Japan \\ ${ }^{3}$ National Institute for Materials Science, Tsukuba 305-0047, Japan \\ ${ }^{4}$ Research Institute of Electrical Communication, Tohoku University, Sendai 980-8577, Japan \\ ${ }^{5}$ London Centre for Nanotechnology and Department of Electronic \& Electrical Engineering, UCL, WC1H OAH, United Kingdom \\ ${ }^{6}$ Center for Science and Innovation in Spintronics, Core Research Cluster, Tohoku University, Sendai 980-8577, Japan
}

(Received 28 January 2020; accepted 29 May 2020; published 19 June 2020)

\begin{abstract}
A perpendicularly magnetized ferromagnetic layer is an important building block for recent/future highdensity spintronic memory applications. This paper reports the fabrication of perpendicularly magnetized $\mathrm{Ni} / \mathrm{Pt}$ superlattices and the characterization of their structures and magnetic properties. The optimization of film growth conditions allowed us to grow epitaxial Ni/Pt (001) superlattices on $\mathrm{SrTiO}_{3}(001)$ single-crystal substrates. We investigated their structural parameters and magnetic properties as a function of the $\mathrm{Ni}$ layer thickness and obtained a high uniaxial magnetic anisotropy energy of $1.9 \times 10^{6} \mathrm{erg} / \mathrm{cm}^{3}$ for a $[\mathrm{Ni}(4.0 \mathrm{~nm}) / \mathrm{Pt}(1.0 \mathrm{~nm})]$ superlattice. In order to elucidate the detailed mechanism on perpendicular magnetic anisotropy for the $\mathrm{Ni} / \mathrm{Pt}$ (001) superlattices, the experimental results were compared with the first-principles calculations. It has been found that the strain effect is a prime source of the emergence of perpendicular magnetic anisotropy.
\end{abstract}

DOI: 10.1103/PhysRevMaterials.4.064413

\section{INTRODUCTION}

A ferromagnetic layer exhibiting room-temperature perpendicular magnetization is an important building block for the development of various spintronic applications [1-9] such as ultrahigh-density magnetic recording devices, magnetic random access memories, and three-terminal spintronic devices. The perpendicularly magnetized state at zero external magnetic field is achieved when a ferromagnetic layer possesses a magnetic anisotropy field in the normal direction to the film plane larger than the demagnetizing field. This perpendicular magnetic anisotropy (PMA) can be obtained by utilizing the bulk magnetic anisotropy and the interface magnetic anisotropy. The metallic superlattices such as $\mathrm{Co} / \mathrm{Pd}$ [10-12], $\mathrm{Co} / \mathrm{Pt}$ [11-13], and $\mathrm{Co} / \mathrm{Ni}$ [14-19] are the materials systems showing clear PMA due to the interface magnetic anisotropy, and several origins for the emergence of interface magnetic anisotropy have been proposed [20]: (i) interface magnetocrystalline anisotropy due to the anisotropic atomic configuration at the interface, which is known as the Néel model, (ii) interface magnetoelastic anisotropy due to the anisotropic strain originating from the lattice mismatch, and (iii) alloying at the interface.

Ni-based metallic superlattices are a representative of PMA originating from a lattice strain, and a recent study has shown electric-field driven switching of magnetization for the $\mathrm{Cu} / \mathrm{Ni}$ multilayers through the magnetoelectric coupling effect [21]. Pioneering works on the magnetic anisotropy for the ultrathin $\mathrm{Ni}[22,23]$ reported that the Ni layer grown on

\footnotetext{
*go-sai@imr.tohoku.ac.jp
}

$\mathrm{Cu}$ (001) was spontaneously magnetized in the perpendicular direction to the film plane at a certain layer thickness. The perpendicular magnetization of the Ni layer was attributable to the strain-induced perpendicular magnetic anisotropy arising from the inverse of magnetostriction [24-28] because of the lattice mismatch between fcc-Cu (001) and fcc-Ni (001) having the lattice constants $a$ of $0.362 \mathrm{~nm} \mathrm{[29]} \mathrm{and}$ $0.352 \mathrm{~nm}$ [30], respectively. According to the studies on the magnetic properties for epitaxial $\mathrm{Cu} / \mathrm{Ni} / \mathrm{Cu}(001)$ sandwiches [31,32], the perpendicular magnetic anisotropy of this system comes from the bulk magnetoelastic anisotropy energy and interface magnetocrystalline anisotropy. The contribution of the former strain-induced effect is a major reason for the wide Ni thickness range exhibiting the spontaneous perpendicular magnetization from 3 to $12.5 \mathrm{~nm}$ [32]. Since the strain plays an important role in the emergence of PMA not only for the $\mathrm{Ni}$ layer on the $\mathrm{Cu}$ substrate but also for the $\mathrm{Ni} / \mathrm{Cu}$ superlattices $[33,34]$, one may expect a larger PMA if a larger lattice strain can be induced in a Ni layer by using the nonmagnetic element having the larger lattice mismatch with $\mathrm{Ni}$ such as fcc-Pt $(a=0.393 \mathrm{~nm})$ [35]. In the 1990s, a few experimental studies reported on the fabrication of (111)-textured $\mathrm{Ni} / \mathrm{Pt}$ superlattices with PMA [36-38]. However, no report had been made for Ni/Pt (001) epitaxial superlattices. Epitaxial or single-crystal-like $\mathrm{Ni} / \mathrm{Pt}$ superlattices are advantageous for the quantitative evaluation of their magnetic properties and the comparison to the theoretical calculations. In addition, taking into account the further investigation of transport and thermoelectric properties of $\mathrm{Ni} / \mathrm{Pt}, \mathrm{Ni} / \mathrm{Pt}$ superlattices should be directly grown on a nonconductive substrate without any buffer layer materials, which is essential to examine the potential of $\mathrm{Ni} / \mathrm{Pt}$ superlattices as spintronic and spin-caloritronic materials [39]. 
In this paper, we report the epitaxial growth of perpendicularly magnetized Ni/Pt (001) superlattices directly on a $\mathrm{SrTiO}_{3}$ single-crystal substrate, which was achieved by optimizing the film growth temperature. We show the structure and magnetic properties for the $\mathrm{Ni} / \mathrm{Pt}$ (001) epitaxial superlattices, in particular the Ni layer thickness dependence of them. These experimental results are compared to the first-principles calculations, from which we are able to reveal that the major origin of the PMA is the strain effect for the Ni/Pt superlattice.

\section{EXPERIMENTAL PROCEDURE}

The layers of $[\mathrm{Ni} / \mathrm{Pt}]_{\times N}$ were grown on a single-crystal substrate employing an ultrahigh vacuum-compatible magnetron sputtering system with the base pressure below $2 \times$ $10^{-7} \mathrm{~Pa}$. We chose the $\mathrm{SrTiO}_{3}$ (100) single-crystal substrate from the viewpoint of lattice matching to the Ni/Pt (001) superlattice. We first deposited the Ni layer on the $\mathrm{SrTiO}_{3}$ substrate followed by the Pt layer. The Ni layer thickness $(t)$ was varied in the range from 1.0 to $6.0 \mathrm{~nm}$ while the $\mathrm{Pt}$ layer thickness was fixed at $1.0 \mathrm{~nm}$. The total thicknesses of superlattices were designed to be approximately $20 \mathrm{~nm}$ by tuning the repetition number $(N)$. The influence of substrate temperature $\left(T_{\mathrm{S}}\right)$ on the layer growth was investigated in order to achieve the epitaxial growth of $[\mathrm{Ni} / \mathrm{Pt}]_{\times N}$ on the $\mathrm{SrTiO}_{3}$ (100) substrate, where $T_{\mathrm{s}}$ was changed in the range from room temperature to $650{ }^{\circ} \mathrm{C}$. The criteria for optimizing $T_{\mathrm{s}}$ are the successful formation of layered structure and the achievement of epitaxial growth for the $\mathrm{Ni}$ and Pt layers. Before starting the deposition experiment, we examined the influence of substrate heating on the insulative characteristics of $\mathrm{SrTiO}_{3}$ substrate because the substrate heating may give rise to the creation of oxygen vacancy and the resultant degradation in the insulative property. We confirmed that the present film growth condition does not affect the properties of $\mathrm{SrTiO}_{3}$ substrate. Finally, using magnetron sputtering, a 2.0-nm-thick Al layer was deposited at room temperature on the $[\mathrm{Ni} / \mathrm{Pt}]_{\times N}$ layers as a capping layer. It is noted that, since a reference sample of $\mathrm{Ni}$ single-layer film with the $\mathrm{Al}$ capping layer did not exhibit the remarkable PMA, the magnetic anisotropy induced at the interface with the $\mathrm{Al}$ capping layer is negligible.

The crystal orientation and the morphology of the layers were monitored by the in situ observation using reflection high-energy electron diffraction (RHEED). Structural characterization was performed using the $\mathrm{x}$-ray diffraction (XRD) with $\mathrm{Cu}-K \alpha$ radiation and transmission electron microscope (TEM) together with the element analysis by the energydispersive X-ray spectroscopy (EDX). Magnetic properties for the thin films were measured using a vibrating sample magnetometer (VSM) and a superconducting quantum interference device (SQUID) magnetometer (MPMS3-SQUID, Quantum Design, Inc.).

\section{EXPERIMENTAL RESULTS AND DISCUSSION}

\section{A. Growth temperature dependence}

Figure 1 displays RHEED images for $[\mathrm{Ni} / \mathrm{Pt}]$ with $t=$ $3.0 \mathrm{~nm}$ and $N=5$, where $T_{\mathrm{s}}$ was set at 200,400 , and $650^{\circ} \mathrm{C}$. The diffraction patterns were observed just after the growth of the fifth Ni layers and the fifth Pt layers. At $T_{\mathrm{S}}=200^{\circ} \mathrm{C}$,

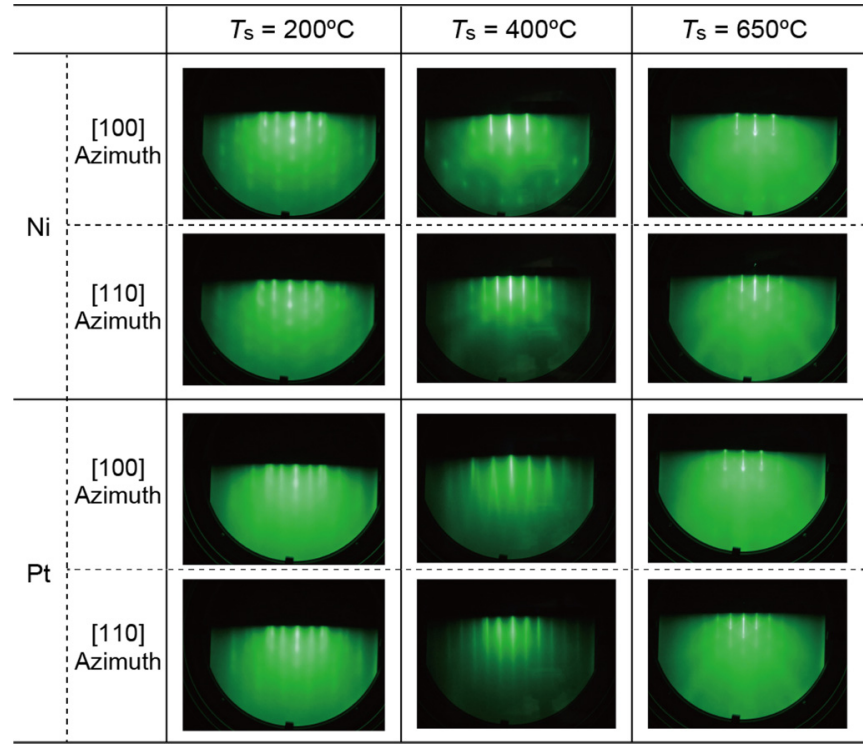

FIG. 1. Reflection high-energy electron diffraction images for $[\mathrm{Ni}(t) / \operatorname{Pt}(1 \mathrm{~nm})]_{\times N}$ with $t=3.0 \mathrm{~nm}$ and $N=5$, where the substrate temperature $\left(T_{\mathrm{s}}\right)$ was set at 200,400 , and $650^{\circ} \mathrm{C}$. The diffraction patterns were observed just after the growth of the fifth Ni layers and the fifth Pt layers.

the spots and the nonperiodic streaks are observed, indicating that the Ni/Pt layers are not epitaxially grown on the $\mathrm{SrTiO}_{3}$ (100) substrate. The diffraction patterns at $T_{\mathrm{s}}=200^{\circ} \mathrm{C}$ also suggest that the preferential crystallographic orientation is the [111] direction for $T_{\mathrm{s}}=200^{\circ} \mathrm{C}$. As $T_{\mathrm{s}}$ is increased to $400^{\circ} \mathrm{C}$, the diffraction patterns are drastically changed and the sharp streaks are observed. These streak patterns may indicate the epitaxial growth of $\mathrm{Ni} / \mathrm{Pt}$ layers with the (001) plane. In addition, RHEED patterns for the fifth Ni layer and the fifth Pt layer exhibited different diffraction patterns, which at least guarantees that intermixing between $\mathrm{Ni}$ and Pt layers is not remarkable at $T_{\mathrm{s}}=400^{\circ} \mathrm{C}$. However, the further increase in $T_{\mathrm{S}}$ leads to the significant intermixing. Both the fifth $\mathrm{Ni}$ layer and the fifth Pt layer grown at $T_{\mathrm{s}}=650^{\circ} \mathrm{C}$ show the similar diffraction patterns even though the streaks become sharper than those for $T_{\mathrm{s}}=400^{\circ} \mathrm{C}$. We consider that $T_{\mathrm{s}}=$ $650^{\circ} \mathrm{C}$ is too high to maintain the layered structure without intermixing. The intermixing at $650^{\circ} \mathrm{C}$ is understood from the phase diagram of Ni-Pt binary alloy [40]. Since the liquidus is located around $1500^{\circ} \mathrm{C}$ for the equiatomic composition of Ni-Pt, $650^{\circ} \mathrm{C}$ is high enough for the induction of atomic diffusion.

The XRD profiles for [Ni/Pt] with $t=3.0 \mathrm{~nm}$ and $N=5$ are shown in Fig. 2(a) for $T_{\mathrm{s}}=200^{\circ} \mathrm{C}$, Fig. 2(b) for $400{ }^{\circ} \mathrm{C}$, and Fig. 2(c) for $650^{\circ} \mathrm{C}$. The reflections of Pt 111 and $\mathrm{Ni}$ 002 have the highest intensities for the samples at $T_{\mathrm{s}}=200$ and $400^{\circ} \mathrm{C}$, respectively, in which the clear satellite peaks appear around the Pt 111 and Ni 200. The appearance of satellite peaks indicates the formation of layered structure. As mentioned above for the RHEED observation, the sample at $T_{\mathrm{S}}=200{ }^{\circ} \mathrm{C}$ is the nonepitaxial Ni/Pt with the (111) preferential orientation and the sample at $T_{\mathrm{s}}=400^{\circ} \mathrm{C}$ is the epitaxial $\mathrm{Ni} / \mathrm{Pt}$ with the (001) orientation. Thus, the appearances of $\mathrm{Pt}$ 111 and $\mathrm{Ni} 002$ for $T_{\mathrm{s}}=200$ and $400{ }^{\circ} \mathrm{C}$, respectively, in the 


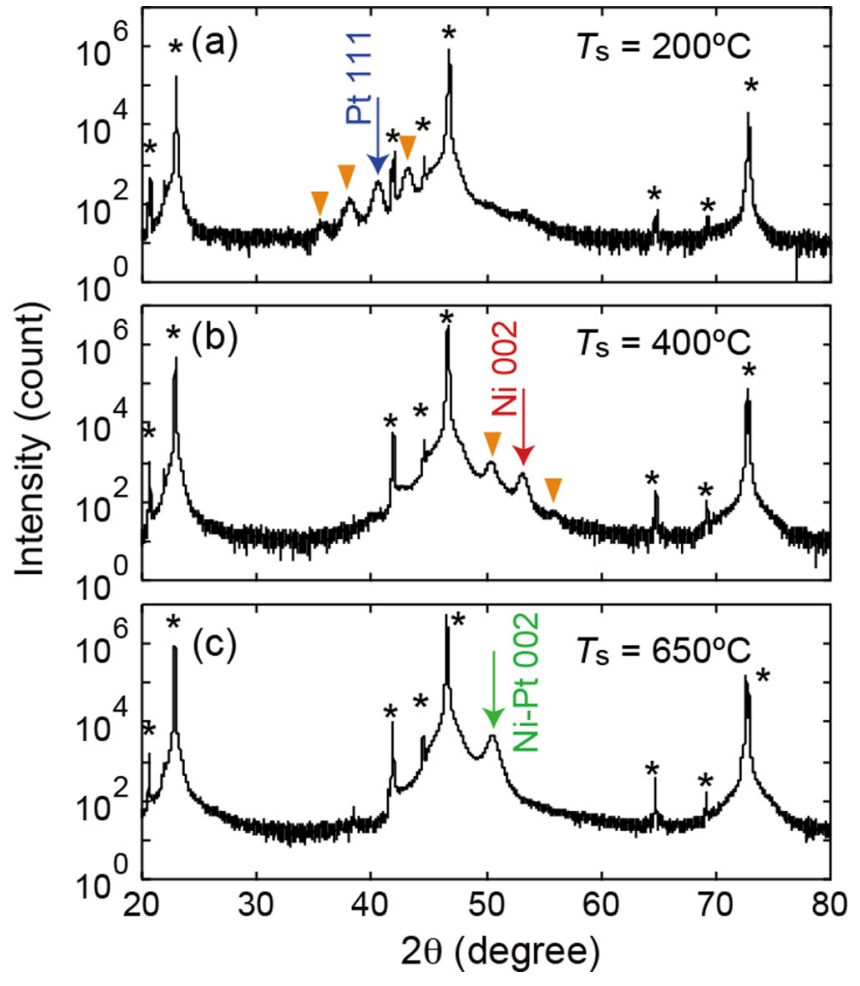

FIG. 2. X-ray diffraction profiles for $[\mathrm{Ni}(t) / \mathrm{Pt}(1 \mathrm{~nm})]_{\times N}$ with $t=3.0 \mathrm{~nm}$ and $N=5$ grown at (a) $T_{\mathrm{s}}=200{ }^{\circ} \mathrm{C}$, (b) $400^{\circ} \mathrm{C}$, and (c) $650{ }^{\circ} \mathrm{C}$. The asterisks denote the reflections from the $\mathrm{SrTiO}_{3}(001)$ substrates. The inverted triangles represent the satellite reflections.

XRD profiles are consistent with the RHEED observation. In this study, however, Ni 111 and Pt 200 reflections were not identified because those peak angles, $2 \theta=44.6^{\circ}$ for Ni 111 and $2 \theta=46.2^{\circ}$ for Pt 200, were overlapped with the large peak from the $\mathrm{SrTiO}_{3}$ substrate. In contrast to the samples at $T_{\mathrm{s}}=200$ and $400^{\circ} \mathrm{C}$, the NiPt 002 appears and no satellite peak is observed at $T_{\mathrm{s}}=650^{\circ} \mathrm{C}$. We consider that alloying was promoted at $T_{\mathrm{s}}=650^{\circ} \mathrm{C}$, which is also consistent with the RHEED observation for $T_{\mathrm{s}}=650^{\circ} \mathrm{C}$.

Figure 3 shows (a) the high-resolution TEM image and (b), (c) EDX mappings for $\mathrm{Ni}$ and $\mathrm{Pt}$ of $[\mathrm{Ni} / \mathrm{Pt}]$ with $t=3.0 \mathrm{~nm}$ and $N=5$ grown at $T_{\mathrm{s}}=400{ }^{\circ} \mathrm{C}$. The initial Ni layer forms islands with the flat surface on the $\mathrm{SrTiO}_{3}(001)$ substrate. This Volmer-Weber growth mode is attributable to the lattice mismatch, which is calculated to be $10 \%$ using $a=0.352 \mathrm{~nm}$ for $\mathrm{Ni}$ and $a=0.391 \mathrm{~nm}$ for $\mathrm{SrTiO}_{3}$, and the wetness of metallic $\mathrm{Ni}$ on the oxide $\mathrm{SrTiO}_{3}$. The subsequent Pt layer starts the layer growth, which covers the $\mathrm{Ni}$ islands. As the layer number is increased, the layered structure becomes well defined. From the structural characterization by RHEED, $\mathrm{XRD}$, and TEM, it is confirmed that the (001) epitaxially grown Ni/Pt superlattice is achieved on the $\mathrm{SrTiO}_{3}(001)$ substrate by optimizing the growth temperature such as $T_{\mathrm{s}}=$ $400{ }^{\circ} \mathrm{C}$.

Figure 4 displays the magnetization curves for the $[\mathrm{Ni} / \mathrm{Pt}]$ with $t=3.0 \mathrm{~nm}$ and $N=5$ grown at (a) $T_{\mathrm{s}}=200^{\circ} \mathrm{C}$, (b) $400{ }^{\circ} \mathrm{C}$, and (c) $650^{\circ} \mathrm{C}$. The red curves denote the magnetization curves measured with in-plane magnetic field (IP curve) while the blue curves denote those measured with
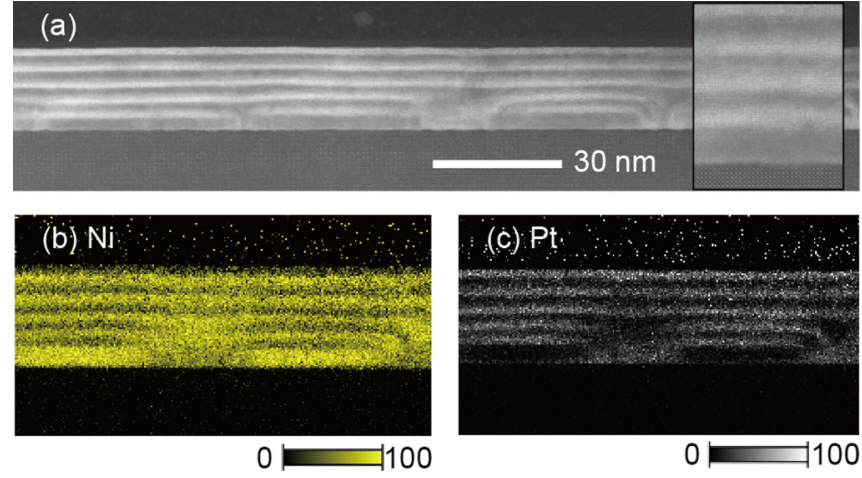

FIG. 3. (a) High-resolution transmission electron microscope image together with (inset) the enlarged image and (b) energydispersive $\mathrm{x}$-ray spectroscopy mappings for $\mathrm{Ni}$ and (c) $\mathrm{Pt}$ of $[\mathrm{Ni}(t) / \mathrm{Pt}(1 \mathrm{~nm})]_{\times N}$ with $t=3.0 \mathrm{~nm}$ and $N=5$ grown at $T_{\mathrm{s}}=$ $400{ }^{\circ} \mathrm{C}$.

out-of-plane magnetic field (OPP curve). The measurements were done at room temperature. In this study, the value of magnetization $(M)$ was defined as the detected magnetic moment per the unit volume of $\mathrm{Ni}$ layers. In the case of $T_{\mathrm{s}}=200^{\circ} \mathrm{C}$, the easy magnetization axis lies in the film plane, which is attributable to the nonepitaxial $\mathrm{Ni} / \mathrm{Pt}$ at $T_{\mathrm{s}}=200^{\circ} \mathrm{C}$. On the other hand, $T_{\mathrm{s}}=400{ }^{\circ} \mathrm{C}$ leads to the high squareness of out-of-plane magnetization curve, indicating that the Ni/Pt

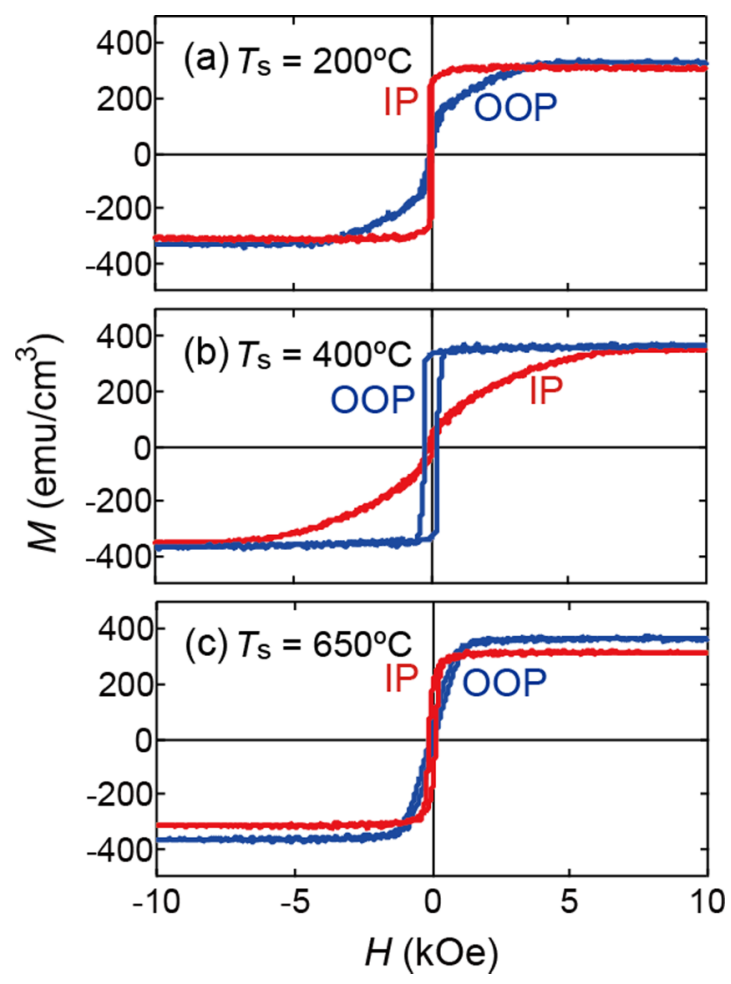

FIG. 4. Magnetization curves for the $[\mathrm{Ni}(t) / \mathrm{Pt}(1 \mathrm{~nm})]_{\times N}$ with $t=3.0 \mathrm{~nm}$ and $N=5$ grown at (a) $T_{\mathrm{s}}=200^{\circ} \mathrm{C}$, (b) $400{ }^{\circ} \mathrm{C}$, and (c) $650^{\circ} \mathrm{C}$. The red curves denote the magnetization curves measured with the in-plane magnetic field (IP) while the blue curves denote those measured with the out-of-plane magnetic field (OPP). The measurement was done at room temperature. 
at $T_{\mathrm{s}}=400^{\circ} \mathrm{C}$ possesses the PMA larger than the shape anisotropy. The further increase in $T_{\mathrm{s}}$ up to $650^{\circ} \mathrm{C}$ gives rise to the in-plane easy magnetization axis again. The disappearance of PMA results from the collapse of layered structure as explained in Fig. 2(c).

In summary, $T_{\mathrm{s}}=400^{\circ} \mathrm{C}$ is the adequate growth temperature for (001)-oriented epitaxial growth as well as the formation of layered structure, leading to the induction of PMA overcoming the shape anisotropy. The origin of PMA will be discussed in Sec. IIIC. Hereinafter, $T_{\mathrm{s}}$ is fixed at $400{ }^{\circ} \mathrm{C}$.

\section{B. Ni layer thickness dependence}

In this subsection, we show the $t$ dependence of structure and magnetic properties of $[\mathrm{Ni} / \mathrm{Pt}]$ grown at $T_{\mathrm{s}}=400^{\circ} \mathrm{C}$, which allows us to reveal the origin of PMA for the (001) epitaxially grown $\mathrm{Ni} / \mathrm{Pt}$ superlattice. Figure 5 shows (a) outof-plane and (b) in-plane XRD profiles for $t=1.5,2.0,3.0$, $4.0,5.0$, and $6.0 \mathrm{~nm}$ together with the profile for the 20.0-nmthick Ni thin film grown on the $\mathrm{SrTiO}_{3}(001)$ substrate. In the out-of-plane XRD profiles, the main reflections come from $\mathrm{Ni}$ 002 and clear satellite peaks are observed. As mentioned in the previous subsection, Pt 200 is not identified because of the overlap with the large substrate peaks. One sees that the $2 \theta$ angles of main and satellite peaks monotonically shift with increasing $t$. As in the case of Ni 002, the Ni 200 reflection seen in the in-plane XRD profiles shows the gradual shift with $t$. From these out-of-plane and in-plane XRD profiles and the RHEED patterns, we find the epitaxial relationship of $(001)_{\mathrm{SrTiO}_{3}}\left\|(001)_{\mathrm{Ni}}\right\|(001)_{\mathrm{Pt}^{2}},[100]_{\mathrm{SrTiO}_{3}}\left\|[100]_{\mathrm{Ni}^{2}}\right\|[100]_{\mathrm{Pt}}$. Figure 6 summarizes (a) the lattice constants of $a$ - and $c$ planes, (b) the value of $c / a$, and (c) the superlattice period $(D)$ as a function of $t$. $D$ was calculated by the following equation: $(2 / \lambda) \sin \theta_{n}=1 / d \pm n / D$ for the $n$th satellite peaks with the x-ray wavelength $(\lambda)$ and the lattice spacing $(d)$. As shown in Fig. 6(a), the value of $a$ is larger than that for bulk Ni [30] whereas the value of $c$ is smaller than that for bulk Ni. These tendencies become remarkable as $t$ is reduced. As a result, the value of $c / a$ is decreased down to 0.90 at $t=1.2 \mathrm{~nm}$. This means that a larger tensile strain exists in the film plane for smaller $t$, and the lattice strain is relaxed as $t$ is increased, leading to the values of $a$ and $c$ approaching to the bulk lattice constant.

Figure 7 shows the magnetization curves for the $[\mathrm{Ni} / \mathrm{Pt}]$ with (a) $t=1.2$, (b) 1.3 , (c) 1.5 , (d) 2.0 , (e) 4.0 , and (f) 5.0 $\mathrm{nm}$, which were measured at room temperature. All the films except for $t=1.2 \mathrm{~nm}$ possess the PMA, resulting in the easy magnetization axis normal to the film plane. The effective uniaxial magnetic anisotropy constant $\left(K_{\text {eff }}\right)$ corresponds to the area enclosed between the OPP and IP curves. As $t$ is increased from 1.3 to $4.0 \mathrm{~nm}$, the saturation field of IP curve gradually increases, indicating the increase in $K_{\text {eff }}$. The further increase in $t$ up to $5.0 \mathrm{~nm}$ gives rise to the reduction of $K_{\text {eff. }}$ The values of $K_{\text {eff }}$, saturation magnetization $\left(M_{\mathrm{s}}\right)$, and uniaxial magnetic anisotropy constant $\left(K_{\mathrm{u}}=K_{\mathrm{eff}}+2 \pi M_{\mathrm{s}}^{2}\right)$ as a function of $t$ are plotted in Figs. 8(a), 8(b), and 8(c), respectively. The value of $K_{\text {eff }}$ is significantly increased in the range of $2.0 \mathrm{~nm} \leqslant t \leqslant 4.0 \mathrm{~nm}$. This means that there exists an adequate $t$ region for enhancing the PMA. $M_{\mathrm{S}}$ is
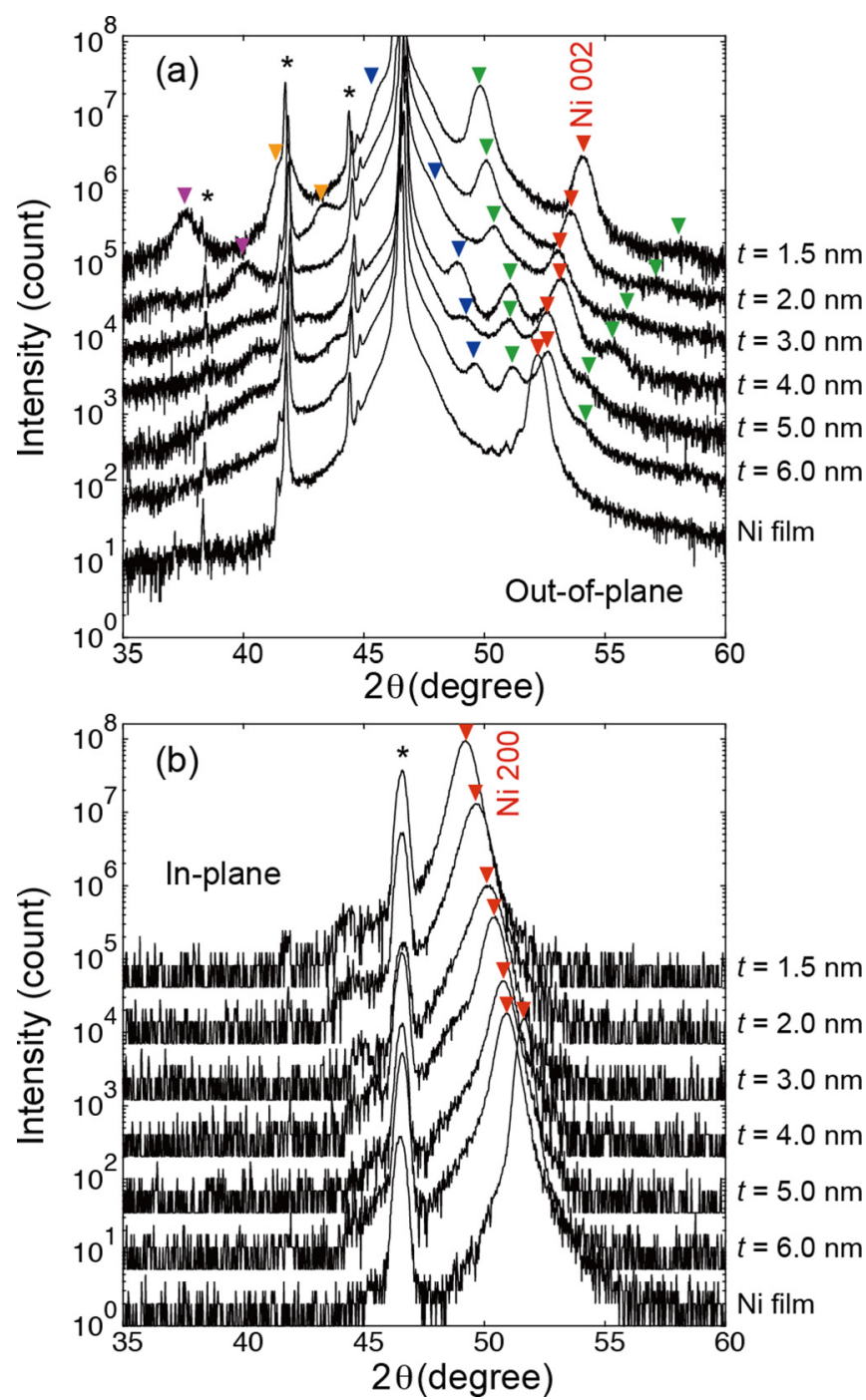

FIG. 5. (a) Out-of-plane and (b) in-plane x-ray-diffraction profiles for the $[\mathrm{Ni}(t) / \mathrm{Pt}(1 \mathrm{~nm})]_{\times N}$ with $t=1.5,2.0,3.0,4.0,5.0$, and $6.0 \mathrm{~nm}$, which were grown at $T_{\mathrm{s}}=400{ }^{\circ} \mathrm{C}$, together with the profile for the 20.0-nm-thick Ni thin film grown on the $\mathrm{SrTiO}_{3}(001)$ substrate. The asterisks denote the reflections from the $\mathrm{SrTiO}_{3}(001)$ substrates. In (a), the inverted triangles except for the red triangles represent the satellite reflections of $\mathrm{Ni} 002$ peaks.

monotonically decreased as $t$ is decreased from 6.0 to $2.0 \mathrm{~nm}$. For example, the value of $M_{\mathrm{s}}$ for $t=4.0 \mathrm{~nm}$ is $380 \mathrm{emu} / \mathrm{cm}^{3}$, which corresponds to $0.46 \mu_{\mathrm{B}}$ using Bohr magneton. This magnetization value is smaller than the bulk $\mathrm{Ni}\left(\sim 0.6 \mu_{\mathrm{B}}\right)$. Below $t=2.0 \mathrm{~nm}, M_{\mathrm{s}}$ is steeply decreased and the sample for $t=1.0 \mathrm{~nm}$ does not exhibit the spontaneous magnetization at room temperature. The $t$ dependence of $K_{\mathrm{u}}$ is similar to that of $K_{\text {eff. }}$. One sees that the [Ni/Pt] samples with $t$ larger than $4.0 \mathrm{~nm}$ also possess the moderate $K_{\mathrm{u}}$. The maximum $K_{\mathrm{u}}$ is $1.9 \times 10^{6} \mathrm{erg} / \mathrm{cm}^{3}$ for $t=4.0 \mathrm{~nm}$.

The remarkable points observed in the $t$ dependence of $K_{\mathrm{u}}$ are the broad thickness region for high $K_{\mathrm{u}}$ and the drastic reduction of $K_{\mathrm{u}}$ below $t=2.0 \mathrm{~nm}$. These features are different from the conventional metallic superlattices showing perpendicular magnetization thanks to the interface magnetic anisotropy, such as $\mathrm{Co} / \mathrm{Pd}$ [10-12], $\mathrm{Co} / \mathrm{Pt}$ [11-13], and $\mathrm{Co} / \mathrm{Ni}$ 

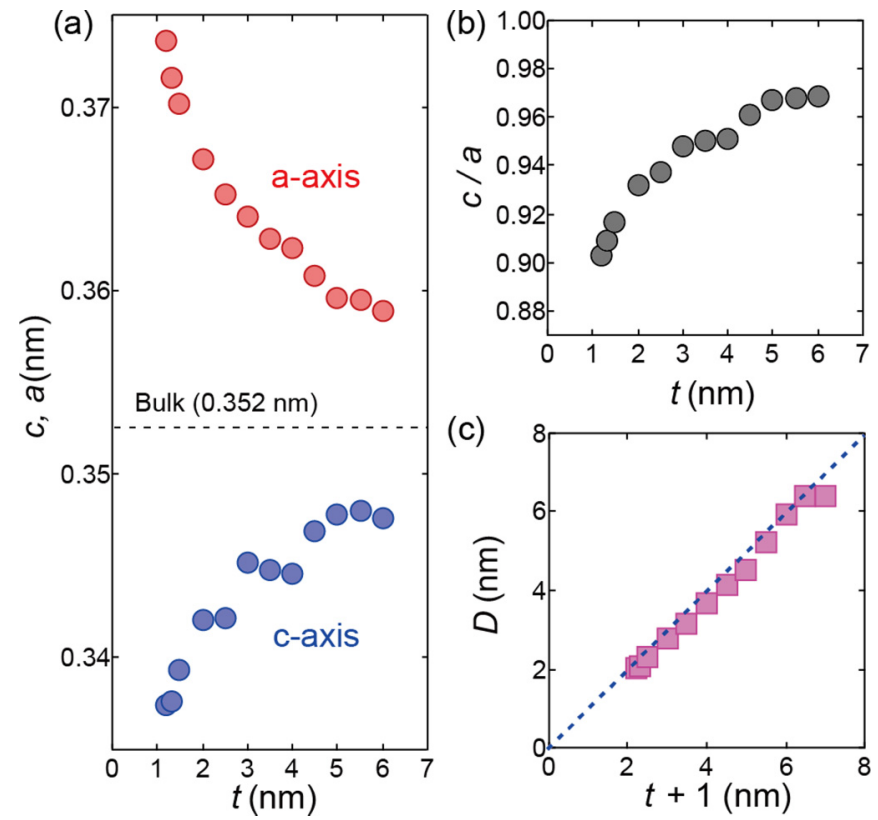

FIG. 6. (a) Lattice constants of $a$ - and $c$ planes, (b) the value of $c / a$, and (c) the superlattice period $(D)$ as a function of $t$ for the $[\mathrm{Ni}(t) / \mathrm{Pt}(1 \mathrm{~nm})]_{\times N}$ grown at $T_{\mathrm{s}}=400{ }^{\circ} \mathrm{C}$.

[14-19]. In contrast to these systems, the broad thickness region for high $K_{\mathrm{u}}$ was reported also for the $\mathrm{Cu} / \mathrm{Ni} / \mathrm{Cu}(001)$ sandwich structures [32]. According to the previous work on the $\mathrm{Ni} / \mathrm{Cu}$ system [32], the strain-dependent magnetic surface
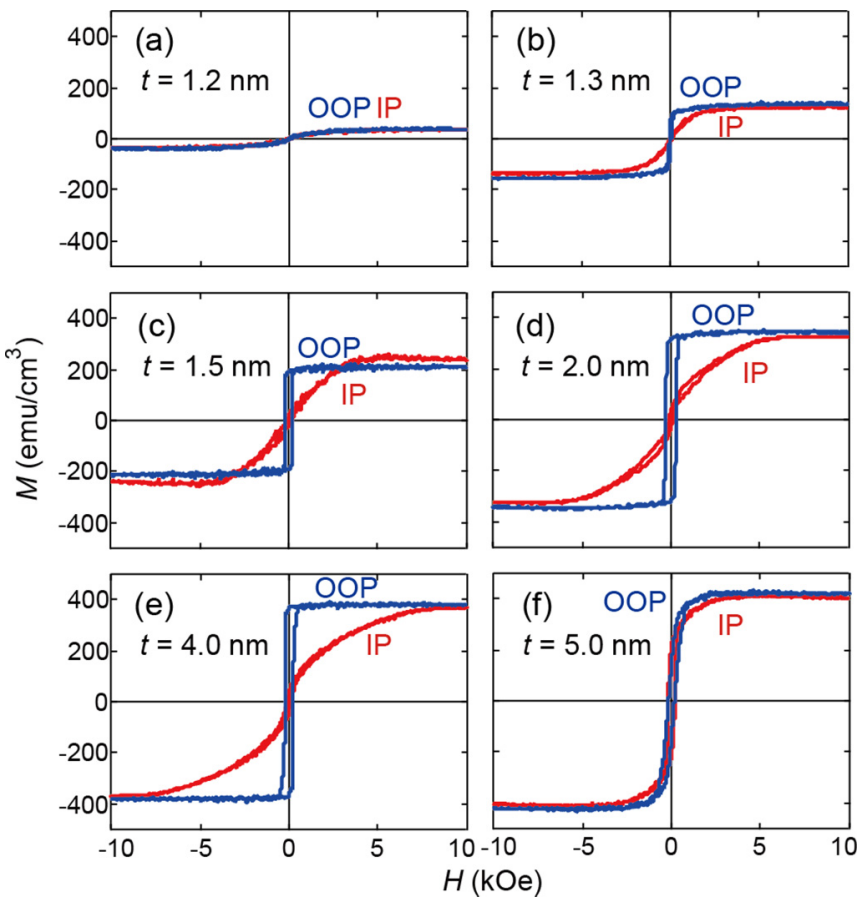

FIG. 7. Magnetization curves for the $[\mathrm{Ni}(t) / \mathrm{Pt}(1 \mathrm{~nm})]_{\times N}$ with (a) $t=1.2$, (b) 1.3 , (c) 1.5 , (d) 2.0 , (e) 4.0 , and (f) $5.0 \mathrm{~nm}$, which were grown at $T_{\mathrm{s}}=400{ }^{\circ} \mathrm{C}$. The red curves denote the magnetization curves measured with the IP while the blue curves denote those measured with the OPP. The measurement was done at room temperature.
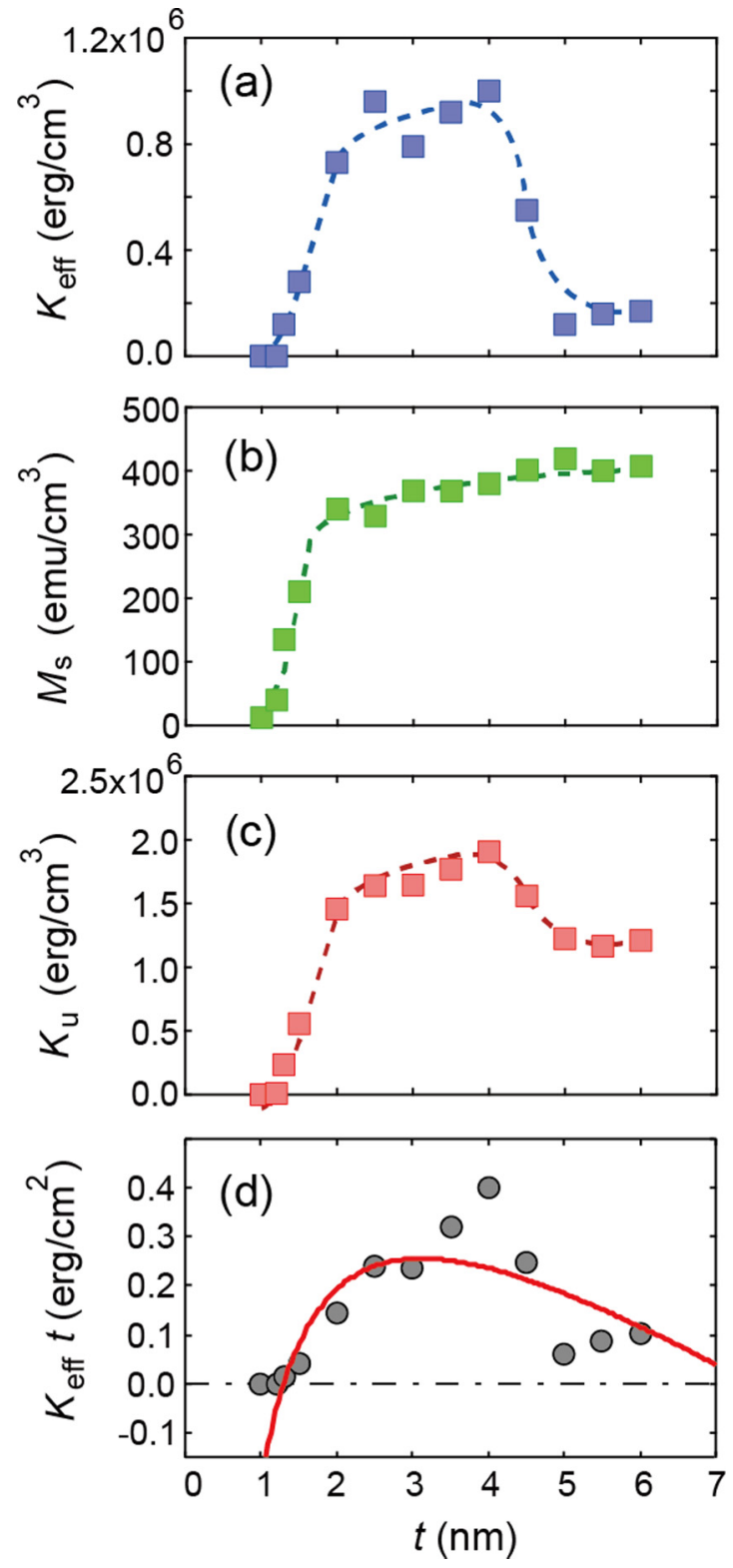

FIG. 8. (a) Effective uniaxial magnetic anisotropy constant $\left(K_{\text {eff }}\right)$, (b) saturation magnetization $\left(M_{\mathrm{s}}\right)$, (c) uniaxial magnetic anisotropy constant $\left(K_{\mathrm{u}}\right)$, (d) $K_{\text {eff }} t$ and as a function of $t$ for the $[\mathrm{Ni}(t) / \mathrm{Pt}(1 \mathrm{~nm})]_{\times N}$ grown at $T_{\mathrm{s}}=400{ }^{\circ} \mathrm{C}$. The solid curve shown in (d) is the result of fitting using Eq. (2).

anisotropy plays a major role for inducing the PMA in the $\mathrm{Ni} / \mathrm{Cu}$ superlattices. In that case, $K_{\text {eff }}$ is phenomenologically described by

$$
K_{\mathrm{eff}}=-2 \pi M_{\mathrm{s}}^{2}+2\left(B_{1}+\frac{B_{\mathrm{s}}}{t}\right) e_{0}(t)+\left(K_{1}+\frac{2 K_{\mathrm{s}}}{t}\right),
$$

where $B_{1}$ is the first-order cubic bulk magnetoelastic coupling coefficient, $B_{\mathrm{S}}$ is the surface magnetoelastic coupling coefficient, $K_{1}$ is the first-order cubic magnetocrystalline anisotropy energy, and $K_{\mathrm{s}}$ is the surface magnetic anisotropy energy. $e_{0}(t)$ represents the average in-plane biaxial misfit strain, which is given by $e_{0}(t)=\eta\left(t_{c} / t\right)$ using the form of the average strain 
[41], where $\eta$ is the film-substrate lattice mismatch and $t_{\mathrm{c}}$ is the thermodynamic critical thickness. With the assumption that $K_{1}$ is negligibly small, Eq. (1) can be transformed into

$$
K_{\mathrm{eff}} t=-2 \pi M_{\mathrm{s}}^{2} t+2\left(B_{1} t_{\mathrm{c}} \eta+K_{\mathrm{s}}\right)+\left(\frac{2 B_{\mathrm{s}} t_{\mathrm{c}} \eta}{t}\right) .
$$

In Fig. 8(d), $K_{\text {eff }} t$ as a function of $t$ is plotted. The experimental data at $t \geqslant 2.0 \mathrm{~nm}$, where the value of $M_{\mathrm{s}}$ keeps the almost constant value of $400 \mathrm{emu} / \mathrm{cm}^{3}$, were fitted with Eq. (2). In Eq. (2), the value of $B_{1}$ also depends on the strain, i.e., thickness-dependent $B_{1}(t)$. The previous works [25-27] mentioned $B_{1}(t)=B_{1}{ }^{\text {bulk }}+D_{1} \varepsilon$, where $B_{1}{ }^{\text {bulk }}$ is $B_{1}$ without strain, $\varepsilon$ is the strain, and $D_{1}$ is the coefficient. In the case of Ni, $B_{1}{ }^{\text {bulk }}=9.4 \times 10^{7} \mathrm{erg} / \mathrm{cm}^{3}$ and $D_{1}=-234 \times$ $10^{7} \mathrm{erg} / \mathrm{cm}^{3}$ were reported [26]. The values of $B_{1}(t)$ for the present $[\mathrm{Ni} \mathrm{Pt}]$ samples are varied in the range from $2.5 \times$ $10^{7} \mathrm{erg} / \mathrm{cm}^{3}$ for $t=2 \mathrm{~nm}$ to $6.5 \times 10^{7} \mathrm{erg} / \mathrm{cm}^{3}$ for $t=6 \mathrm{~nm}$. Therefore, we fitted the $t$-dependent $K_{\text {eff }} t$ using two values of $B_{1}=2.5 \times 10^{7} \mathrm{erg} / \mathrm{cm}^{3}$ and $6.5 \times 10^{7} \mathrm{erg} / \mathrm{cm}^{3}$. In this study, we assumed the value of $\eta$ as $\eta\left(a_{\mathrm{f}}-a_{\mathrm{s}}\right) / a_{\mathrm{s}}$ with the lattice constants of film $\left(a_{\mathrm{f}}\right)$ and substrate $\left(a_{\mathrm{s}}\right)$. If $\eta$ is calculated using the bulk lattice constants of $\mathrm{Ni}(a=0.352 \mathrm{~nm})$ and Pt $(a=0.393 \mathrm{~nm}), \eta$ is obtained to be 0.1 . In this case, the Pt layers are regarded as a very solid layer like a substrate. However, this idea may not be appropriate because the lattice constant of $\mathrm{Pt}$ is also influenced by the formation of interface with the Ni layers. This means that the actual value of $\eta$ must be much lower than 0.1. Unfortunately, it is difficult to strictly determine the value of $\eta$. Then, based on the previous study on $\mathrm{Ni} / \mathrm{Cu}$ [32], in which $\eta$ was set at 0.026 , we fitted the present experimental result with $\eta=0.026$ and $t_{\mathrm{c}}=1.8 \mathrm{~nm}$. As a result, $K_{\mathrm{s}}=0.32 \pm 0.04 \mathrm{erg} / \mathrm{cm}^{2}\left(K_{\mathrm{s}}=0.14 \pm 0.04 \mathrm{erg} / \mathrm{cm}^{2}\right)$ and $B_{\mathrm{s}}=-10.4 \pm 3.0 \mathrm{erg} / \mathrm{cm}^{2}$ were obtained for $B_{1}=2.5 \times$ $10^{7} \mathrm{erg} / \mathrm{cm}^{3}\left(B_{1}=6.5 \times 10^{7} \mathrm{erg} / \mathrm{cm}^{3}\right)$. Those are of the same order as the values reported for the $\mathrm{Ni} / \mathrm{Cu}$ system [32]. Because of the uncertainty of $\eta$ as discussed above, it is hard to quantitatively discuss the values of $K_{\mathrm{s}}$ and $B_{\mathrm{s}}$. That uncertainty also may be a reason why the steep change in $K_{\text {eff }} t$ at $4.0 \mathrm{~nm} \leqslant t \leqslant 5.0 \mathrm{~nm}$ is not reproduced by the calculation. As an effective way to solve the uncertainty of $\eta$, we consider that the empirical expression for the strain taking the stress into account would be helpful [27]. We however emphasize that Eq. (2) qualitatively explains the experimental tendency, which strongly suggests that the strain effect, i.e., the value of $B_{1}$, largely contributes to the emergence of PMA rather than $K_{\mathrm{s}}$. In the next subsection, the effect of the lattice strain on the magnetic anisotropy will be discussed based on the comparison between the experimental results and theoretical calculation.

Although the increased $K_{\mathrm{u}}$ in the thickness range of $2.0 \mathrm{~nm} \leqslant t \leqslant 4.0 \mathrm{~nm}$ is attributable to the strain effect, the lattice strain cannot explain the remarkable reduction of $K_{\mathrm{u}}$ below $t=2.0 \mathrm{~nm}$ because the Ni lattice is significantly distorted even at $t<2.0 \mathrm{~nm}$. In Fig. 8, one may be aware that the reduction of $K_{\mathrm{u}}$ is accompanied by the reduction of $M_{\mathrm{s}}$. In order to understand the reason for the remarkable reduction of $M_{\mathrm{S}}$ at $t<2.0 \mathrm{~nm}$, we measured the measurement temperature (T) dependence of $M$ for $t=1.0,1.5,2.0,3.0$, and $4.0 \mathrm{~nm}$ as

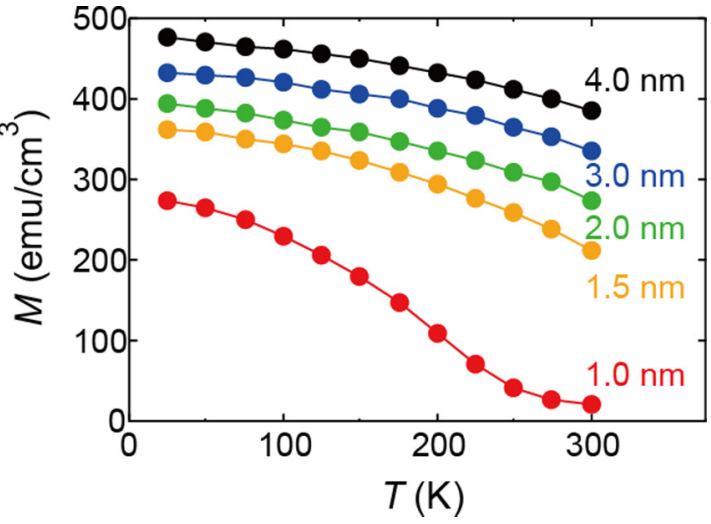

FIG. 9. Measurement temperature $(T)$ dependence of magnetization $(M)$ for the $[\mathrm{Ni}(t) / \mathrm{Pt}(1 \mathrm{~nm})]_{\times N}$ with $t=1.0,1.5,2.0,3.0$, and $4.0 \mathrm{~nm}$, which were grown at $T_{\mathrm{s}}=400{ }^{\circ} \mathrm{C}$.

shown in Fig. 9. These $M-T$ curves suggest the Curie temperature is gradually decreased with decreasing $t$ and becomes lower than room temperature for $t=1.0 \mathrm{~nm}$. Consequently, we find that the remarkable reduction of $M_{\mathrm{S}}$ at $t<2.0 \mathrm{~nm}$ originates from the decrease in the Curie temperature for the thin Ni layers.

\section{Theoretical calculations}

In this subsection, the effect of the lattice strain on the magnetic anisotropy is discussed based on the first-principles calculation results. The first-principles calculations were performed by using the Vienna Ab initio Simulation Package [42] with the generalized gradient approximation parametrized Perdew, Burke, and Ernzerhof [43] and projector-augmented wave potentials [44]. The wave functions were expanded in a plane-wave basis setup to a cutoff kinetic energy of $500 \mathrm{eV}$. The cell volume and all of atomic positions were relaxed within the constraint of the fixed in-plane lattice constant.
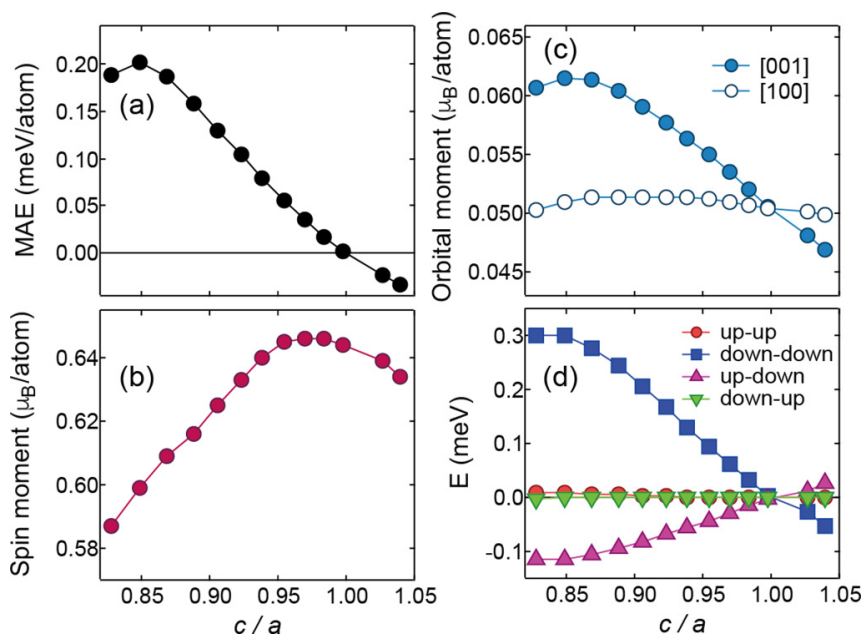

FIG. 10. (a) Magnetic anisotropy energy (MAE), (b) spin moment, (c) orbital moments for [001] and [100] directions, and (d) energies $(E)$ for the spin-conserving process (up-up or down-down) and the spin-flip process (up-down or down-up) as a function of $c / a$ for the bulk Ni. 
(a) $\mathrm{Ni}(17$ monolayers)

Pt (17 monolayers)

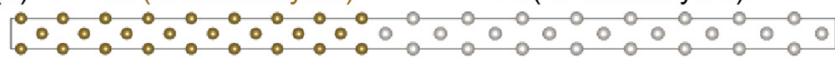

(b)

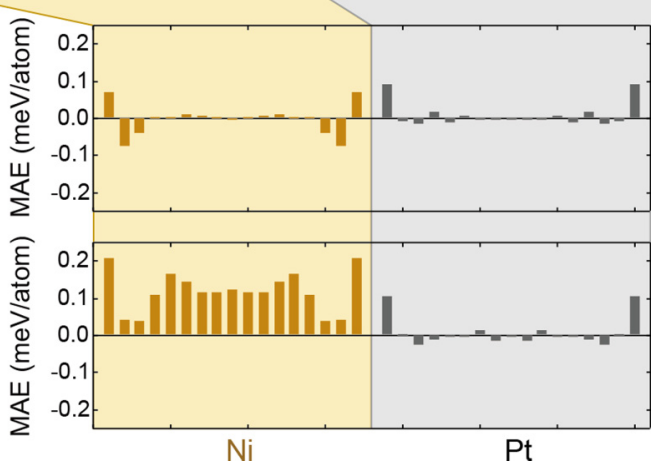

FIG. 11. (a) Schematic illustration of model for the firstprinciples calculation, which consists of $\mathrm{Ni} 17$ monolayers and $\mathrm{Pt}$ 17 monolayers. (b) Position dependence of MAE calculated with the in-plane lattice constant of $a=0.352 \mathrm{~nm}$ and (c) $a=0.372 \mathrm{~nm}$.

The magnetic anisotropy energy (MAE) was obtained using the magnetic force theorem method. The $24 \times 24 \times 24$ and $24 \times 24 \times 1 k$-point mesh were used for the evaluation of the MAE for the Ni bulk and the multilayer consisting of $\mathrm{Ni}$ 17 monolayers (MLs) and Pt 17 MLs. First, we examined the MAE for the Ni bulk induced by the tetragonal lattice distortion as shown in Fig. 10(a). In the present calculation, the positive MAE means that the easy magnetization axis lies along the $c$ axis of distorted $\mathrm{Ni}$. The positive MAE is induced for the Ni bulk at $c / a<1$, i.e., by the in-plane tensile strain. The MAE is proportional to the orbital moment anisotropy for the $3 d$ transition metals as derived by Bruno [45]. The orbital magnetic moment is increased as the tensile strain is induced while the spin magnetic moment is decreased [see Figs. 10(b) and $10(\mathrm{c})$ ]. One sees that the orbital magnetic moment magnetized along the [001] direction is enhanced by the tensile strain as shown in Fig. 10(c), resulting in the induction of PMA. In order to obtain the insight of PMA, we estimated the MAE contribution from the second-order perturbation of the spin-orbit coupling [see Fig. 10(d)] [45,46]. The PMA mainly comes from the spin-conserving term between the minority spin states whereas the spin-flipping contribution is negligible for the MAE of Ni bulk.
Next, we calculated the MAE for the Ni/Pt interface. The layer-resolved MAE for the Ni/Pt interface is shown in Fig. 11, where the in-plane lattice constant of $\mathrm{Ni}$ matches that of Pt. Figure 11(a) illustrates the model of calculation, and Figs. 11(b) and 11(c) are the position dependence of MAE calculated with the in-plane lattice constant of $a=$ $0.352 \mathrm{~nm}$ and $a=0.372 \mathrm{~nm}$, respectively. The interfacial Ni and Pt layers show the PMA regardless of the in-plane lattice parameter. However, the second and third Ni MLs away from the interface exhibit the in-plane magnetic anisotropy in the case of in-plane lattice constant for the Ni bulk [Fig. 11(b)]. Figure 12 shows the local density of states for the $\mathrm{Ni}$ atom in Ni/Pt multilayer. At the interfacial $\mathrm{Ni}$ atom, $d_{x^{2}-y^{2}}$ and $d_{x y}$ states exist below and above the Fermi level, respectively, and the spin-orbit coupling matrix element of $\left\langle d_{x^{2}-y^{2}}\left|\ell_{z}\right| d_{x y}\right\rangle$ contributes to the PMA. For the second and third layers, on the other hand, $d_{3 z^{2}-r^{2}}$ state is increased near the Fermi level compared to $d_{x^{2}-y^{2}}$ state, and the matrix element of $\left\langle d_{3 z^{2}-r^{2}}\left|\ell_{x}\right| d_{x z}\right\rangle$ contributes to in-plane magnetic anisotropy. For the multilayer with the in-plane lattice constant of $\mathrm{Pt}$ bulk [Fig. 11(c)], all the Ni layers from the 4th to 14th ML show the large PMA induced by the tensile tetragonal distortion. The PMA of the Ni/Pt superlattice is attributed to both the non-negligible interfacial contribution and the major bulk contribution induced by lattice distortion. This is different from the other Ni-based superlattices such as $\mathrm{Ni} / \mathrm{Au}$ and $\mathrm{Ni} / \mathrm{Pd}$, in which the interfacial contribution is negative, i.e., $K_{\mathrm{s}}<0$, or negligibly small [20]. As mentioned in the analysis of Fig. 8(d), we have found that the strain effect largely contributes to the emergence of PMA for the $\mathrm{Ni} / \mathrm{Pt}$ superlattices. At the same time, we experimentally evaluated the non-negligible interface magnetic anisotropy energy. Therefore, the above first-principles calculation results are qualitatively consistent with the experimental results.

\section{SUMMARY}

We investigated the optimum film growth conditions to achieve the epitaxial growth of the perpendicularly magnetized Ni/Pt (001) superlattices directly on the $\mathrm{SrTiO}_{3}$ (001) single-crystal substrate. We found that $T_{\mathrm{s}}=400{ }^{\circ} \mathrm{C}$ was the adequate growth temperature for (001)-oriented (a)

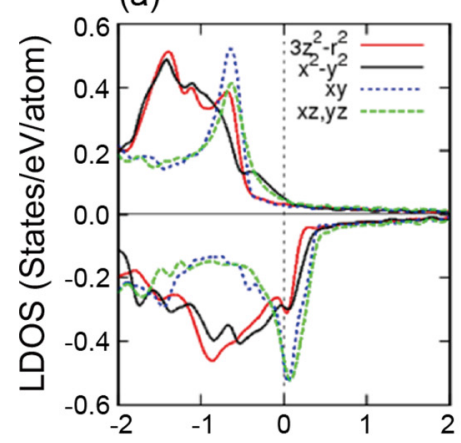

(b)

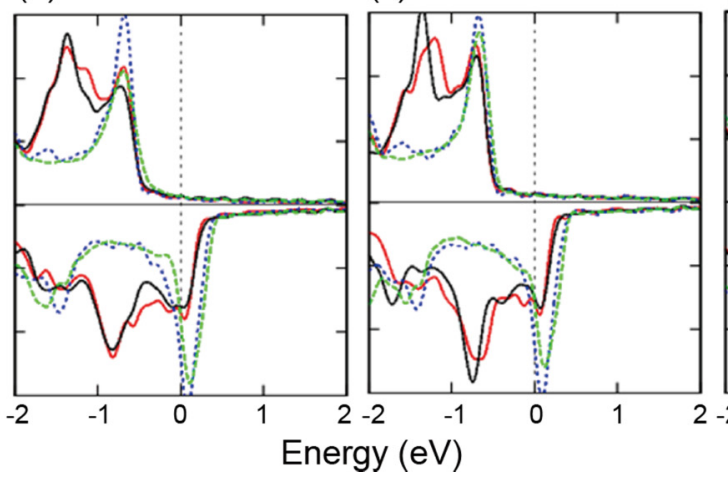

(d)

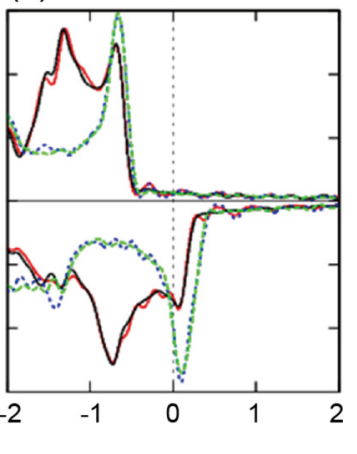

FIG. 12. (a) Local density of states for first, (b) second, (c) third, and (d) ninth Ni atomic layer from the Ni/Pt interface, where the in-plane lattice constant was set at $a=0.352 \mathrm{~nm}$. 
epitaxial growth as well as the formation of layered structure. This (001)-oriented epitaxial growth induced the PMA overcoming the shape anisotropy, resulting in the perpendicularly magnetized $\mathrm{Ni} / \mathrm{Pt}$. We obtained the high $K_{\mathrm{u}}=$ $1.9 \times 10^{6} \mathrm{erg} / \mathrm{cm}^{3}$ for $t=4.0 \mathrm{~nm}$. The Ni layer thickness dependence of structural parameters and magnetic properties clearly indicated that the strain effect largely contributes to the emergence of PMA. This experimental finding was supported by the first-principles calculation. The first-principles calculation also suggested the non-negligible contribution of interface magnetic anisotropy to the PMA, which was qualitatively consistent with the experimental results. The findings in this study will provide the useful knowledge for developing a perpendicularly magnetized superlattice.

\section{ACKNOWLEDGMENTS}

The authors thank T. Kubota and Y. Sakuraba for their valuable comments. The structural characterization was partly carried out at the Cooperative Research and Development Center for Advanced Materials, IMR, Tohoku University. This work was supported by the Grant-in-Aid for Scientific Research (S) from JSPS KAKENHI, Japan (Grant No. JP18H05246) and the GIMRT Program of the Institute for Materials Research, Tohoku University (Proposal No. 19K0506).
[1] A. D. Kent and D. C. Worledge, Nat. Nanotechnol. 10, 187 (2015).

[2] S. S. P. Parkin and S.-H. Yang, Nat. Nanotechnol. 10, 195 (2015).

[3] S. Mangin, D. Ravelosona, J. A. Katine, M. J. Carey, B. D. Terris, and E. E. Fullerton, Nat. Mater. 5, 210 (2006).

[4] T. Seki, S. Mitani, K. Yakushiji, and K. Takanashi, Appl. Phys. Lett. 88, 172504 (2006).

[5] T. Seki, S. Mitani, and K. Takanashi, Phys. Rev. B 77, 214414 (2008).

[6] H. Yoda, T. Kishi, T. Nagase, M. Yoshikawa, K. Nishiyama, E. Kitagawa, T. Daibou, M. Amano, N. Shimomura, S. Takahashi, T. Kai, M. Nakayama, H. Aikawa, S. Ikegawa, M. Nagamine, J. Ozeki, S. Mizukami, M. Oogane, Y. Ando, S. Yuasa et al., Curr. Appl. Phys. 10, e87 (2010).

[7] S. Ikeda, K. Miura, H. Yamamoto, K. Mizunuma, H. D. Gan, M. Endo, S. Kanai, J. Hayakawa, F. Matsukura, and H. Ohno, Nat. Mater. 9, 721 (2010).

[8] I. M. Miron, K. Garello, G. Gaudin, P. J. Zermatten, M. V. Costache, S. Auffret, S. Bandiera, B. Rodmacq, A. Schuhl, and P. Gambardella, Nature (London) 476, 189 (2011).

[9] W. Zhou, T. Seki, H. Arai, H. Imamura, and K. Takanashi, Phys. Rev. B 94, 220401(R) (2016).

[10] P. F. Carcia, A. D. Meinhaldt, and A. Suna, Appl. Phys. Lett. 47, 178 (1985).

[11] P. F. Carcia, J. Appl. Phys. 63, 5066 (1988).

[12] F. J. A. den Broeder, W. Hoving, and P. J. H. Bloemen, J. Magn. Magn. Mater. 93, 562 (1991).

[13] C.-J. Lin, G. L. Gorman, C. H. Lee, R. F. C. Farrow, E. E. Marinero, H. V. Do, H. Notarys, and C. J. Chien, J. Magn. Magn. Mater. 93, 194 (1991).

[14] G. H. O. Daalderop, P. J. Kelly, and F. J. A. den Broeder, Phys. Rev. Lett. 68, 682 (1992).

[15] F. J. A. den Broeder, H. W. van Kesteren, W. Hoving, and W. B. Zeper, Appl. Phys. Lett. 61, 1468 (1992).

[16] V. M. Naik, S. Hameed, R. Naik, L. Pust, L. E. Wenger, G. L. Dunifer, and G. W. Auner, J. Appl. Phys. 84, 3273 (1998).

[17] S. Girod, M. Gottwald, S. Andrieu, S. Mangin, J. McCord, E. E. Fullerton, J.-M. L. Beaujour, B. J. Krishnatreya, and A. D. Kent, Appl. Phys. Lett. 94, 262504 (2009).

[18] A. Shioda, T. Seki, J. Shimada, and K. Takanashi, J. Appl. Phys. 117, 17C726 (2015).
[19] T. Seki, J. Shimada, S. Iihama, M. Tsujikawa, T. Koganezawa, A. Shioda, T. Tashiro, W. Zhou, S. Mizukami, M. Shirai, and K. Takanashi, J. Phys. Soc. Jpn. 86, 074710 (2017).

[20] W. J. M. de Jonge, P. J. H. Bloemen, and F. J. A. den Broeder, in Ultrathin Magnetic Structures I, edited by J. A. C. Bland and B. Heinrich (Springer, Berlin, Heidelberg, 1994), pp. 65-85.

[21] Y. Shirahata, R. Shiina, D. L. González, K. JA Franke, E. Wada, M. Itoh, N. A. Pertsev, S. van Dijken, and T. Taniyama, NPG Asia Mater. 7, e198 (2015).

[22] W. L. O’Brien and B. P. Tonner, Phys. Rev. B 49, 15370 (1994).

[23] B. Schulz and K. Baberschke, Phys. Rev. B 50, 13467 (1994).

[24] W. L. O’Brien, T. Droubay, and B. P. Tonner, Phys. Rev. B 54, 9297 (1996).

[25] D. Sander, Rep. Prog. Phys. 62, 809 (1999).

[26] Th. Gutjahr-Löser, D. Sander, and J. Kirschner, J. Appl. Phys. 87, 5920 (2000).

[27] D. Sander, J. Phys.: Condens. Matter 16, R603 (2004).

[28] D. Sander, W. Pan, S. Ouazi, J. Kirschner, W. Meyer, M. Krause, S. Müller, L. Hammer, and K. Heinz, Phys. Rev. Lett. 93, 247203 (2004)

[29] M. Ellner, K. Kolatschek, and B. Predel, J. Less-Common Metals 170, 171 (1991).

[30] M. Nuding and M. Ellner, J. Alloys Compd. 252, 184 (1997).

[31] R. Jungblut, M. T. Johnson, J. aan de Stegge, A. Reinders, and F. J. A. den Broeder, J. Appl. Phys. 75, 6424 (1994).

[32] G. Bochi, C. A. Ballentine, H. E. Inglefield, C. V. Thompson, and R. C. O’Handley, Phys. Rev. B 53, 1729(R) (1996).

[33] R. Naik, A. Poli, D. McKague, A. Lukaszew, and L. E. Wenger, Phys. Rev. B 51, 3549 (1995).

[34] Y. Shirahata, E. Wada, M. Itoh, and T. Taniyama, Appl. Phys. Lett. 104, 032404 (2014).

[35] A. Gibaud, M. Topic, G. Corbel, and C. I. Lang, J. Alloys Compd. 484, 168 (2009).

[36] M. Angelakeris, P. Poulopoulos, N. Vouroutzis, M. Nyvlt, V. Prosser, S. Visnovsky, R. Krishnan, and N. K. Flevaris, J. Appl. Phys. 82, 5640 (1997).

[37] S.-C. Shin, G. Srinivas, Y.-S. Kim, and M.-G. Kim, Appl. Phys. Lett. 73, 393 (1998).

[38] Y.-S. Kim and S.-C. Shin, Phys. Rev. B 59, 6597(R) (1999).

[39] K. Uchida, T. Kikkawa, T. Seki, T. Oyake, J. Shiomi, Z. Qiu, K. Takanashi, and E. Saitoh, Phys. Rev. B 92, 094414 (2015). 
[40] P. Nash and M. F. Singleton, in Binary Alloy Phase Diagrams, 2nd ed., edited by T. B. Massalski, Vol. 3 (AMS, Materials Park, OH, 1990), p. 2841.

[41] C. Chappert and P. Bruno, J. Appl. Phys. 64, 5736 (1988).

[42] G. Kresse and J. Hafner, Phys. Rev. B 47, 558 (1993).
[43] J. P. Perdew, K. Burke, and M. Ernzerhof, Phys. Rev. Lett. 77, 3865 (1996).

[44] P. E. Blöchl, Phys. Rev. B 50, 17953 (1994).

[45] P. Bruno, Phys. Rev. B 39, 865 (1989).

[46] Y. Miura, S. Ozaki, Y. Kuwahara, M. Tsujikwa, K. Abe, and M. Shirai, J. Phys.: Condens. Matter 25, 106005 (2013). 